\title{
Terrien's Disease: Clinical and Ultrastructural Studies, Five Case Reports
}

\author{
Y. POULIQUEN, P. DHERMY, G. RENARD, L. GOICHOT-BONNAT, G. FOSTER \\ AND M. SAVOLDELLI \\ Paris
}

\begin{abstract}
Summary
Terrien's disease occurs in middle-aged patients and is characterised by an insidious thinning of the cornea near the limbus. In most cases, this results in a peripheral ectasia associated with a severe degree of astigmatism. Inflammatory signs are rarely observed in this marginal corneal degeneration which is of unknown aetiology. Electron and light microscopic studies have been performed on five specimens from penetrating keratoplasties. Anatomical and clinical correlations showed the marked marginal degenerations of the corneal stroma to be consistently associated with lipid deposits, but without inflammatory cell infiltrate. These changes are in agreement with previous reported pathological descriptions of Terrien's disease.
\end{abstract}

Terrien's disease is a rare corneal affection. It was first described by Terrien ${ }^{15}$ is 1900 under the name of 'Marginal, symmetrical, ectatic corneal dystrophy'. Clinical and histological descriptions ${ }^{5,6,7,10,17}$ made it possible to distinguish several clinical varieties of the disease.

Terrien's disease consists of a peripheral corneal thinning which leads to ectasia after a prolonged interval. Several radial vessels are found in the thinned relatively transparent area, the anterior margin of which is marked with a dense yellowish area suggesting lipid deposits. In most cases, the disease affects both corneae, is symmetrical and most often observed in men over 60 years old, the condition progresses very slowly, but the thinning and ectasia can still continue for several years and is often associated with an severe astigmatism. No inflammatory signs can be observed, but the pain and redness which have occasionally been reported have been related to puberty and menstrual cycles. ${ }^{1}$
Sometimes, spontaneous perforation can necessitate urgent treatment.

Previous electron microscopic studies have been reported, but these have only been performed in lamellar corneal buttons. ${ }^{9}$ These studies attempt to subdivide the disease into two groups: quiescent and inflammatory, however, the aetiology or pathogenicity of the condition is still unknown. The five cases reported here have been subject to both light and transmission electron microscopic studies: the diagnosis was made initially on Terrien's described criteria.

\section{Case Reports}

Case No. 1 (Fig. 1, Panel I)

MBG, a 42 years old, tool-maker, presented in April 1983 with progressive visual loss. His visual acuity was less than $1 / 10$ in both eyes, N14 in the right eye and $\mathrm{N} 5$ in the left eye. Both eyes had an against the rule astigmatism of more than six dioptres: the acuity of the right eye could not be 

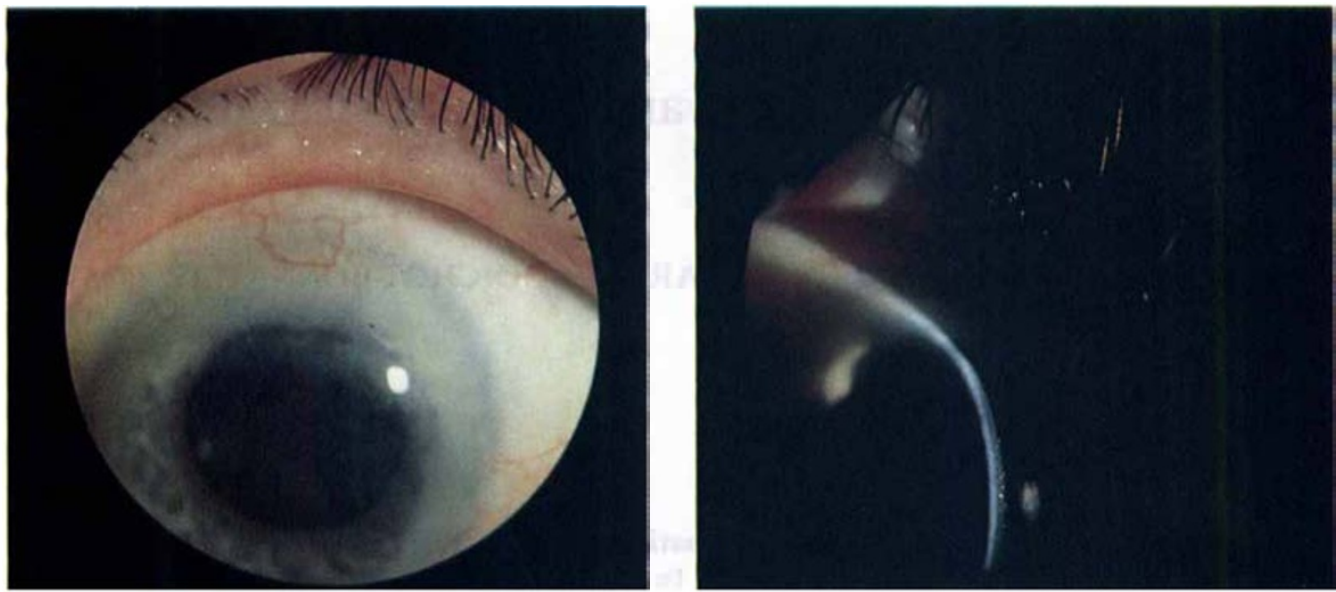

Figs. 1 and 2. Case 1: MBC: 42. Male, Right Eye, Flattening of the corneal curvature in its vertical axis. Marked peripheral thinning is observed at 12 o'clock, leading to a crescent shaped ectasia. Deep corneal opacities can be seen in this ectatic area which is crossed over by fine vessels.
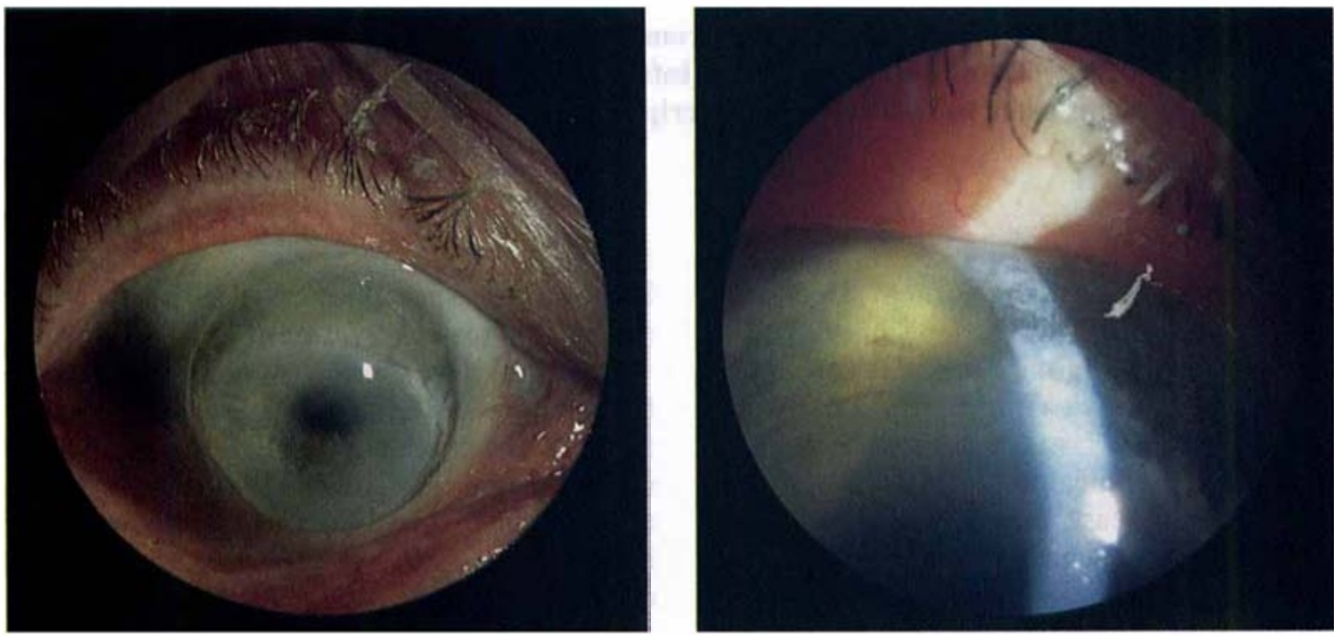

Figs. 3 and 4. Case 4: de St Mar: 77. Male, Advanced bilateral Terrien's disease. The central corneal protrusion is surrounded by severe perilimbal thinning. Its inner border is limited by a yellow ring, which corresponds to fatty deposits.

\section{Panel I}

improved, but the left was improved to $4 / 10$ with -6 D.Sph, at $90^{\circ}$. Slit lamp examination revealed a flattening of the corneal curvature in its vertical axis. A marked peripheral annular thinning was observed at 12 o'clock leading to a crescent shaped ectasia extending from three to nine o'clock. Deep corneal opacities were observed in this area which was covered by fine vessels.

The rest of the ocular examination was normal in this alcoholic patient.

On September 5, 1984, we performed a $9 \mathrm{~mm}$ penetrating keratoplasty, decentered at 12' o'clock and passing through the limbus to enclose the ectactic area. The specimen was fixed for pathological examination. There was no intra-operative complication. On April 27, 1985, the graft was transparent after a period of post-operative oedema; the astigmatism was +3 D.sph at $90^{\circ}$, the visual acuity was $1 / 10$ and N2, the number of endothelial cells was 900 per square millimetre. The continuous suture was removed because of loosened loops. The visual acuity was less than expected considering the clar- 
ity of the donor and button. An optic nerve neuropathy due to smoking and alcoholism may explain this poor visual result.

\section{Case No. 2}

Mrs O M, from Martinique was a 74 years old insulin dependent diabetic with only one eye,

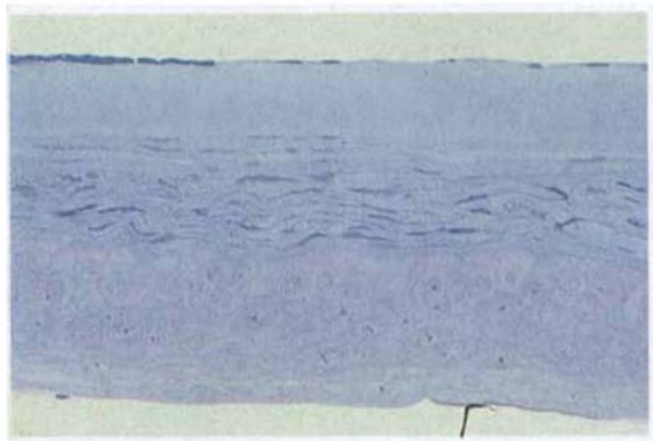

Fig. 1. Case 2: Perilimbal corneal thinning $(0.12 \mathrm{~mm})$. The Epithelium is thick, Bowman's membrane is missing. The corneal stroma is thin but quite normal in its lamellar organisation.

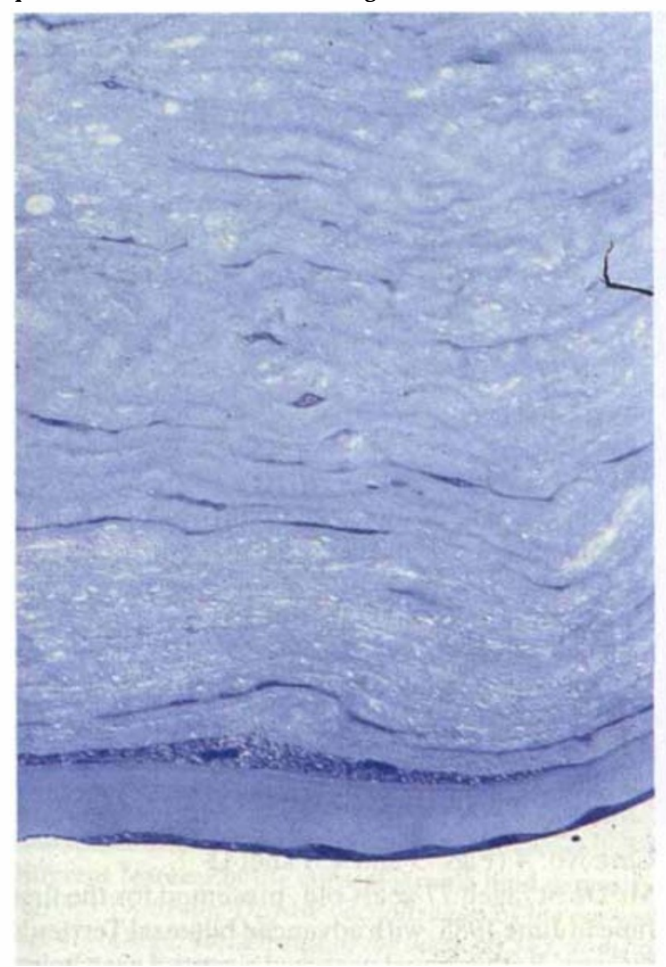

Fig. 2. Case 2: In a thicker zone: Vacuolated stroma, and thick Descemet's membrane. Endothelial cells are present. which had been lost following injury at the age of 32 years. At 47, she felt the first visual signs in her right eye. Corneal deformation developed progressively until 1981 when she was sent to our hospital. She was found to have a typical superior Terrien's marginal ectasia characterised by a corneal thinning between three and nine o'clock, corneal vessels and

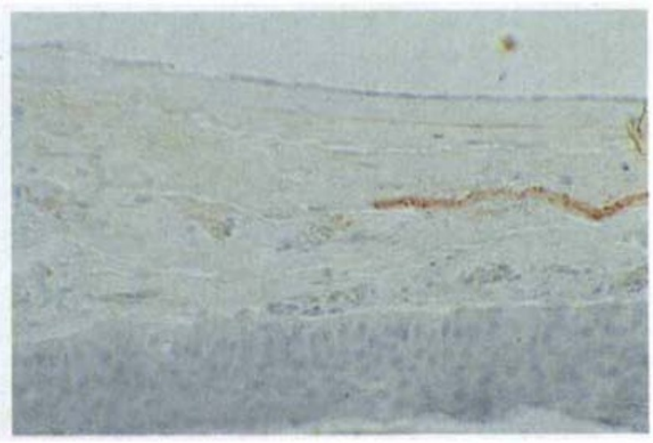

Fig. 3. Case 3: Soudan III staining. A positive demonstration of lipidic deposits.

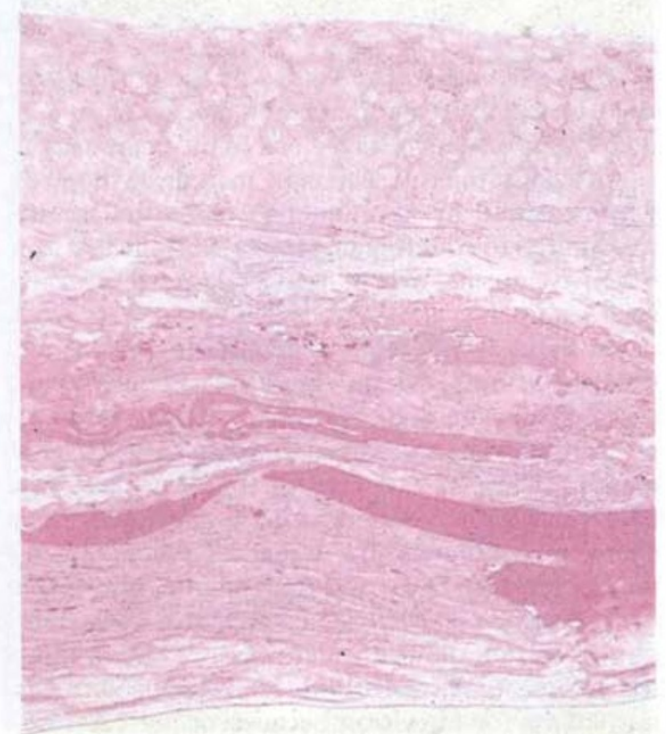

Fig. 4. Case 4: P.A.S. staining. The stromal connective tissue and the vacuolated corneal cells take up the stain. 


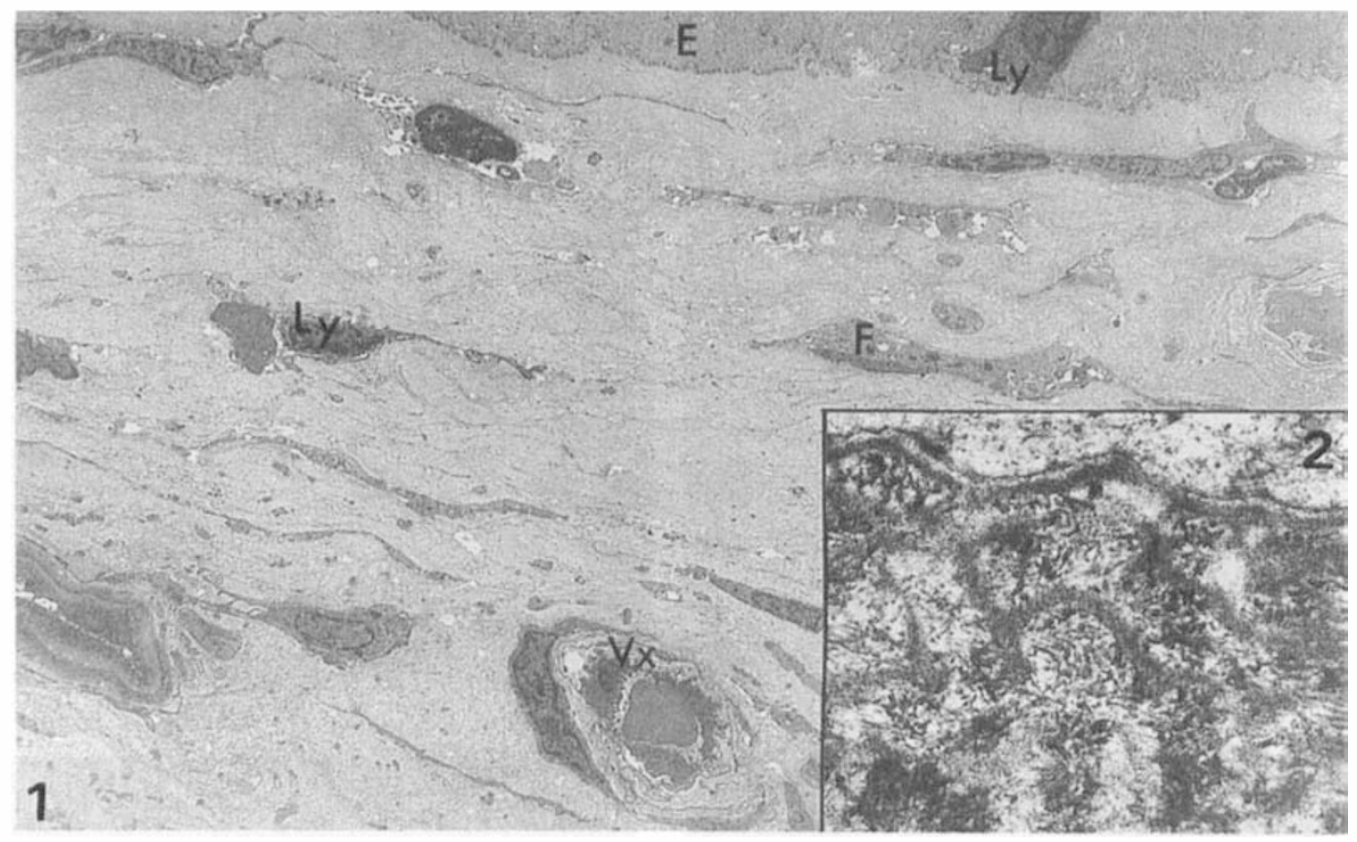

Fig. 1 (TEM $\times 2400)$. Case 1: Sub Epithelial zone. Some lymphocytes $(L y)$ are seen between basal epithelial cells $(E)$ and are also present in the anterior stroma. Somme vessels $(V x)$ are running into this thin zone of the perilimbal cornea.

Fig. 2. (TEM $\times 31950)$. Case 1: Abnormal basal lamina: Duplicated, disrupted and abnormal collagen fibrils.

\section{Panel III}

yellowish deposits. The centre of the cornea was clear. A small circular descemetocele of $2 \mathrm{~mm}$ diameter was located at six o'clock in the ectatic area.

An against the rule astigmatism of three dioptres was measured by keratometry. The visual acuity was limited to counting finger at $1.5 \mathrm{~m}$, the eye was otherwise normal except for a slight posterior capsular cataract.

On February 26, 1981 a penetrating half circle superior corneo-scleral keratoplasty was performed between four and eight o'clock and the resected specimen was fixed for pathological examination. The superior area of the transplant was recovered with conjunctiva. The post-operative course was unremarkable except for a slight inflammatory reaction of the anterior chamber during the first few days, which was accompanied by a transient elevation of intraocular pressure. She left for Martinique on April 10,1981. Her visual acuity was unchanged for far vision because of her cataract, but she could read large characters for near vision. The anterior chamber was quiet, the central cornea was clear, the corneal button well fitting and the ocular pressure was normal. The patient has not been seen since.

Case No. 3

Mr B A, 64 years old, complained 20 years ago of a transient, short lived episodic pain and redness of his right eye. Terrien's disease was diagnosed. The lesion was roughly symmetrical consisting of marginal corneal thinning of the inferior nasal area of his right eye with some vascularisation. Severe astigmatism limited the visual acuity to counting fingers at one metre. There was an inferior thinning in the left eye with a minor deformation which permitted a visual acuity of $7 / 10$. Two months later, although a penetrating keratoplasty had been programmed for the right eye, a perforation took place in the thinned zone.

A penetrating $9 \mathrm{~mm}$ keratoplasty was performed which was decentered towards the sclera to include the perforated area. Six months later, the patient had cataract surgery in the same eye, following which the corneal button became cloudy. The patient has not been seen since.

\section{Case No. 4 (Figs. 3 and 4, Panel I)}

$\mathrm{Mr}$ De St, aged 77 years old, presented for the first time in June 1985, with advanced bilateral Terrien's disease. Both corneal protused a central area being of normal thickness surrounded by severe perilimbal thinning. This area was transluscent, markedly thin, ectactic and was invaded by small vessels. Its inner border was limited by a yellowish ring. 


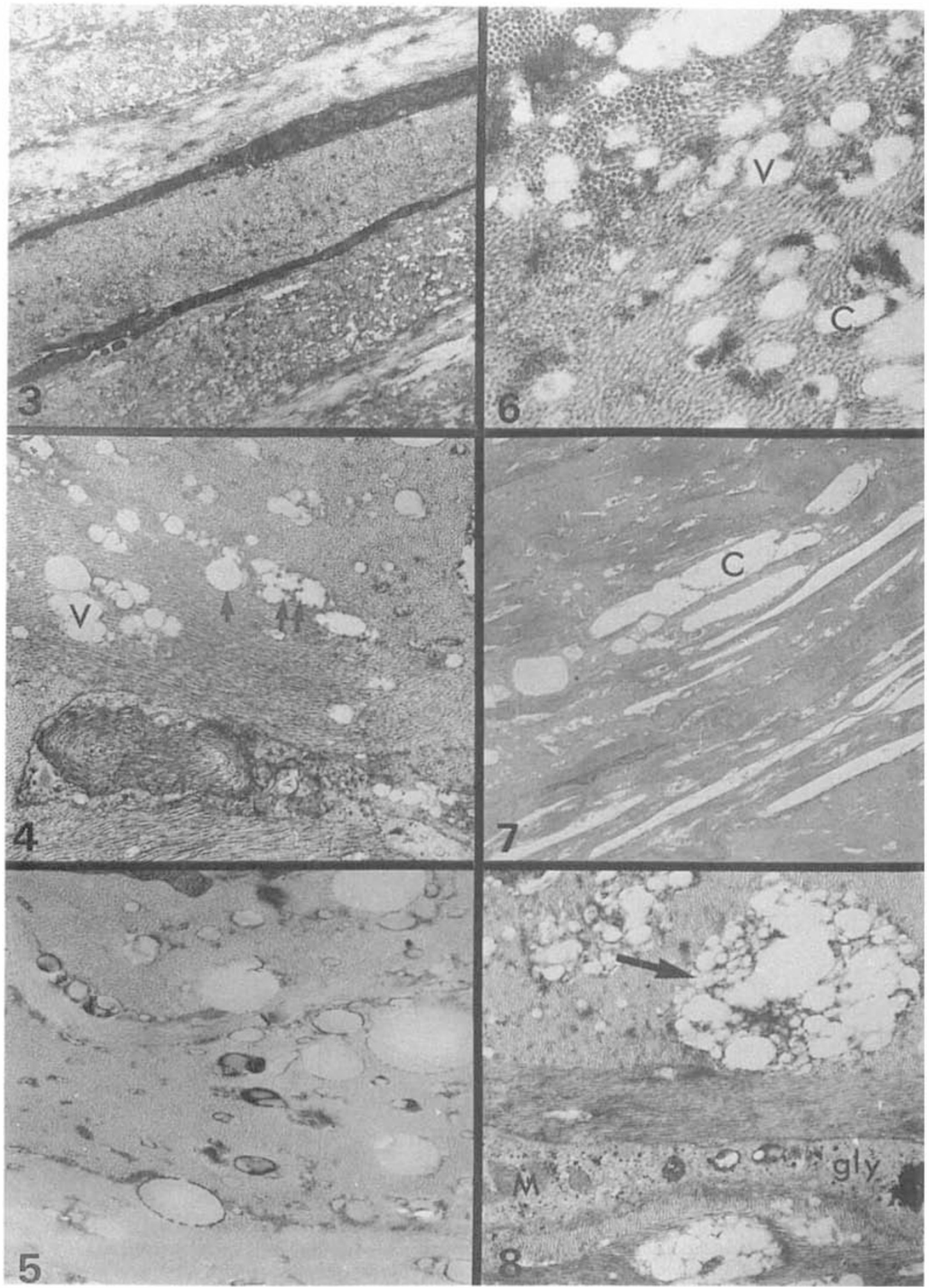

Different features of the corneal stroma lipid deposits.

Fig. 3. $(\times 6140)$ : Diffuse vacuolization of the corneal lamellae.

Fig.4. $(\times 10740):$ Details of the vacuoles $(V)$, some of them are surrounded by a thin dark membrane (arrows).

Fig. 5. $(\times 13150)$ : Lipidic coloration of the vacuolar deposits.

Fig. 6. $(\times 24120)$ : Vacuoles $(V)$ crystal $(C)$.

Fig. 7. $(\times 2310)$ : Lipid crystal $(C)$.

Fig. 8. (× 10740): Grouped vacuoles in the stroma. Note the keratocyte activity: mitochondriae $(M)$ and glycogen (Gly) deposits, in the cytoplasm of the cell. 


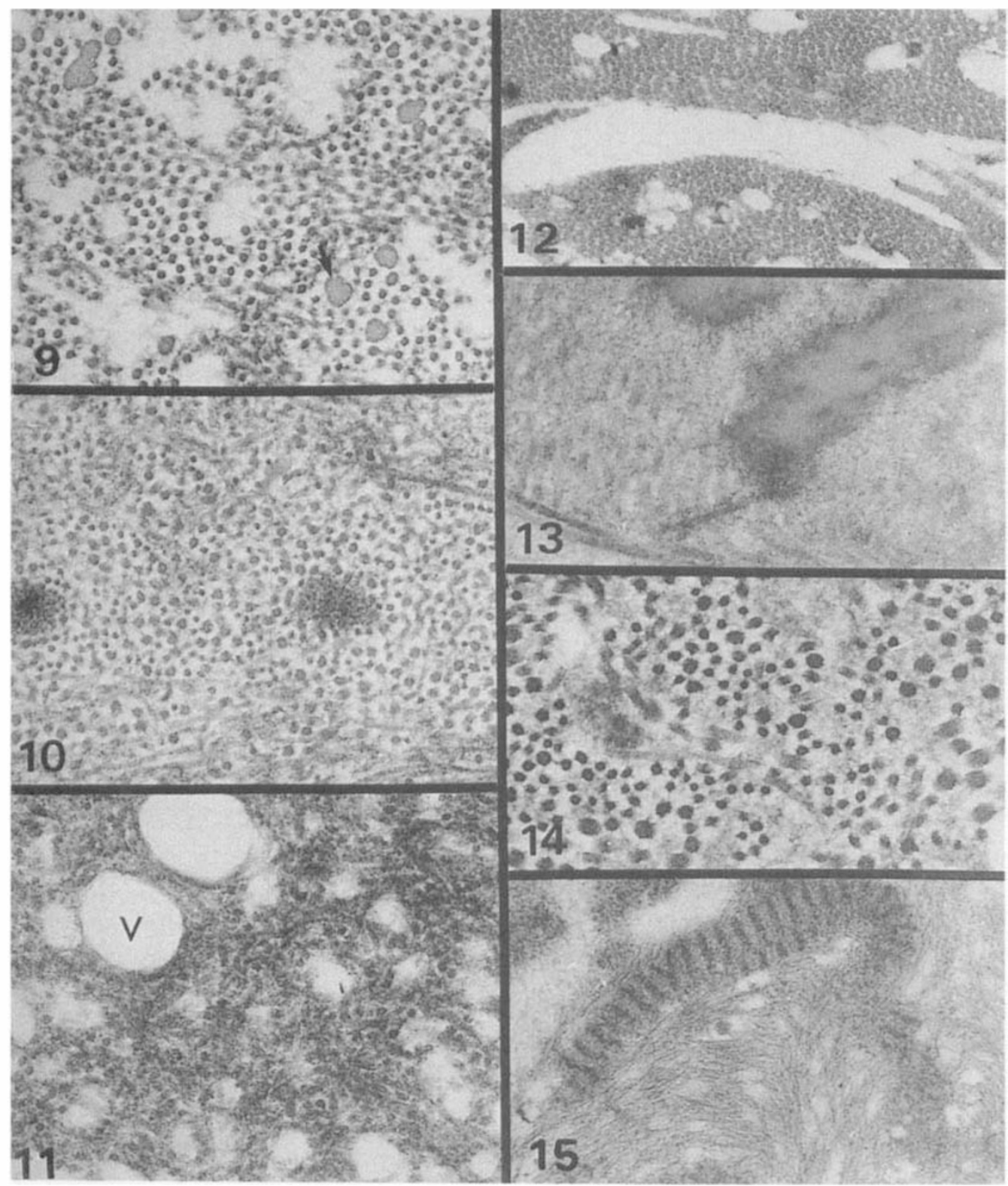

Fig. 9. ( $\times 39460):$ Vacuolar diffuse infiltration of the stroma. Collagen fibrillar degneration (arrows).

Fig. 10. ( $\times 39460)$ : Grouped microfibrils, dispersed collagen fibrills whose diameter is heterogenous.

Fig. 11. ( $\times 39460)$ : Fibrinoid degeneration of the collagen fibrills around the lipid vacuoles.

Fig. 12. $(\times 24120)$ : In the neighbouring of the cholesterol crystals collagen fibrills are modified.

Fig. 13. $(\times 394690):$ Elastin deposits in the stroma.

Fig. 14. ( $\times$ 39460): Corneal scar tissue formation.

Fig. 15. $(\times 24120)$ : Long period collagen formation in the thinned part of the cornea.

\section{Panel V}


Table I

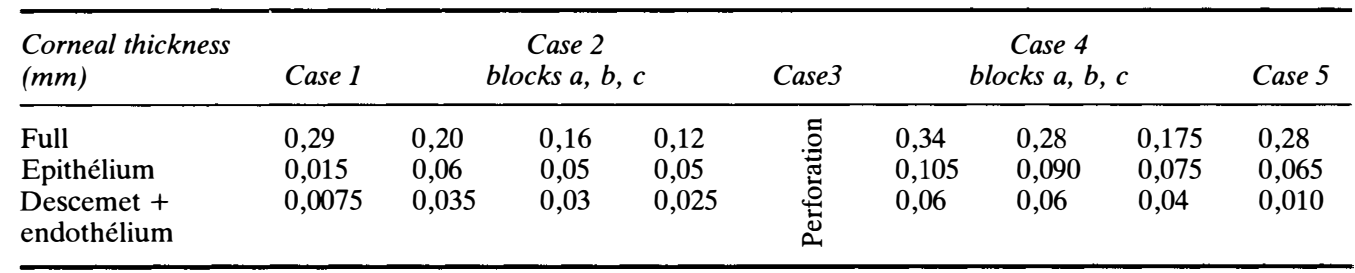

Normal thickness for central cornea is $0,45 \mathrm{~mm}$, and peripheral cornea 0,65 , after

The astigmatism was so severe that it could not be measured, the visual acuity was $1 / 20$, reading was impossible. The marginal deformation was more marked in the left eye than in the right. A moderate nuclear cataract was severe in both eyes. The intraocular pressure was normal.

On October 11, 1985, a penetrating keratoplasty of $11 \mathrm{~mm}$ was performed on the right eye. After a perilimbal conjunctival dissection, the trephination allowed removal of the central cornea with its thinned periphery. The patient was treated with dexamethasone and cyclosporin A. Six months later the corneal button was still clear. The visual acuity was 2/10 and N4 despite the presence of an against the rule astigmatism of six dioptries. In April 1986, there was a small allograft reaction which lead to an opacification of the graft.

In May 1986, a penetrating keratoplasty of $11 \mathrm{~mm}$ was performed on the left eye, combined with an extracapsular cataract extraction with a posterior intraocular lens implantation. The corneal graft remained clear for 18 months under a regimen of dexamethasone and cyclosporin $\mathrm{A}$. Later, this also became cloudy after an allograft reaction. The patient's vision was still $1 / 10$ in the right eye which permitted him to move around. The two corneas were subjected to a light and electron microscopic study.

\section{Case No. 5}

Mr B J, 36 years old, presented with progressive astigmatism leading to severe cornea plana in the vertical axis of the left eye such that the vision could not be improved. A similar though less severe condition affected the right eye, in which visual acuity was 5/10. The left eye which was seriously affected, presented with a typical appearance with marked thinning of the cornea between six and nine o'clock. This thinning was discontinuous consisting of an apposition of small thinned areas surrounded by yellowish deposits and normal limbus areas. The patient's history revealed episodic discrete redness which did not require any anti-inflammatory treatment. Otherwise, the patient was in good health.

In July 1986, a penetrating keratoplasty of $9 \mathrm{~mm}$ was performed in the left eye, it was decentered at six o'clock (the specimen was fixed for pathological examination). One year later, the visual acuity was $5 / 10$ and p 2 despite the presence of a slight corneal oedema in the inferior half of the corneal button. Local corticotherapy was maintained continuously.

\section{Materials and Methods}

All surgical specimens had the same treatment: half the tissue fixed in a Bouin's solution, embedded in paraffin and stained with hematoxoline-eosine for light microscopic examination the other half was treated for electron microscopic examination. This was fixed in a glutaraldehyde solution $(2,5 \%)$ for two hours, washed out in a Sorensen buffer solution pH 7.4, dehydrated in alcohol baths of increasing concentrations, embedded in epon, and cut in medium and ultra thin sections. Cases 4 and 5 specimens were placed in formol for lipidic study and treated in a similar fashion for lipid electron microscopic examination.

\section{Results}

\section{Light microscopic examination}

(Table I, Figs. 1, 2, 3, 4, Panel II)

This confirmed a severe thinning of the perilimbal area. This thinning was different from one case to another and from one area to another in the same specimen. Epithelial changes and thickness irregularities were noticed in the four non perforated cases: epithelium was normal (case 1), thickened (cases 4 and 5), or much thinned (case 2). The cells were stratified and normal except in certain areas where rare inflammatory cells were found. In these same areas, Bowman's membrane was absent, fragmented or broken. The underlying corneal stroma was modified in all its layers (cases 4, 5) and especially in its anterior (case 1) or posterior (case 2) layers. 
Changes were also noticed in the shape of the fibrocytes which were distended or modified adjacent to the anomalies of the collagen fibres. They also conserved their vacuoled cytoplasm. Few inflammatory cells were found in the stroma in all the cases.

Blood vessels were constantly observed. Descemet's membrane was either normal or slightly altered being thickened with posterior tuberosities (case 2), sometimes it was broken or healed by posterior fibrous tissue (case 4). A few endothelial cells were always present in the posterior surface of Descemet's membrane. The perforated cornea in case 3 did not differ from the others especially in central area: cholesterol crystals were particularly evident there, being scattered within the stromal lamella and around 'spumous' cells surrounded by inflammatory cells. Soudan III staining used in cases 4 and 5 revealed a dense lipidic infiltration.

\section{Electron microscopic examination}

The epithelium present in the thinned area was never completely normal. In cases 1,3 and 4 it was stratified with basal, intermediate and superficial cells. The interstitial spaces were dilated and filled with a granular material which was more or less dense to electrons (cases 1 and 3). Sometimes, optically clear vacuoles were found in basal and intermediate cells (case 3 ). In case 2 , the epithelium in certain areas was thinned and infiltrated with inflammatory cells (cases 2 and 3 ). Some of these cells also contained large, numerous, irregular mitochondria.

The basal lamina which was always altered, thickened, discontinuous, broken or duplicated was lying on an altered stroma. Most of the time, hemidesmosomes adhesions were observed (Figs. 1, 2, Panel III).

Bowman's membrane was either broken or absent. Some of its fragments could be identified in the anterior altered layers of the corneal tissue (cases 2, 3, 4 and 5). In cases 2 and 5 , Bowman's membrane was present, in contact with the epithelium, but, presented some important modifications: fragments of basal membrane were superimposed and interlaced with small microfibrillar islands. Sometimes these fragments were condensed into dark irregular patches containing clear vacuolated spaces which were not surrounded by any cell membrane. In case 4 , membrane residues were found at a distance from the epithelium.

The thinned corneal stroma presented numerous modifications; such as a lamellar structural disorganisation. Certain lamellae had a good collagen fibre arrangement while others were totally disorganised, their collagen fibres being scattered in different directions.

Generally, the stroma/lamellae were disorganised with small cavities, optically clear, circular or oval shaped. Some of the vacuoles seemed to have no membrane, while others seemed to be lined with a discontinuous dense material, others also were delimited by a continuous cytoplasmic cellular-like membrane. Some of the vacuoles contained more or less confluent pieces of membrane.

In case 3 these vacuoles, similar to those observed above, were scattered within large lipidic crystals with which they were sometimes in contact and were often associated with electron dense deposits. These could be arranged in rings around the vacuoles, gathered in small island at a distance, or even scattered within the lamella (Figs 3-8, Panel IV).

The fine granular material in these dense deposits was often in close contact with the lipid crystal in case 3 . In case 1 , the represent the most important anomaly.

Scattered elastin and fibrin fibres were found in the stroma of cases 1 and 4 . In case 4 , irregular patches of long periodicity collagen fibres were scattered within the fibrocytes and the fragment of Descemet's membrane.

Certain areas in the stroma showed signs of healing reconstruction which consisted of a heterogenicity of the collagen fibres. Elsewhere collagen fibres degeneration was characterised by the absence of contact between fibrillar and interfibrillar space or deposits. This degeneration is usually accompanied by fibrinoid collagen degeneration (Figs. 9-15, Panel V).

Stroma fibroblasts cells had different appearances according to the area examined. They were usually abnormal, often intensively activated, or even subjected to severe changes (case 1) such as cellular necrosis. The severity of the changes was proportional to corneal vacularisation. 
They were generally activated with a glycogen rich cytoplasm and developed organelles. Numerous cells possessed optically clear vacuoles.

In case 3 , numerous macrophages were grouped within lipid crystals in infiltrated areas where many spumous cells were found. It was among these cells that plasma cells were found. Specific lipidic staining was positive in electron microscopic specimens of cases 4 and 5.

Numerous vacuole cells containing microfibrillar material were observed in case 4 as well as some polymorphonuclear cells. Inflammatory cells were rare, but were found particularly in two specimens. In case 3, they were located near the perforation and areas with intensive lipid infiltrate; in case 4 they were found near the residue of Descemet's membrane and under the epithelium.

Numerous blood vessels and nerve fibres were found in all the specimens. Descemet's membrane was either broken (case 3) or fragmented with different pieces scattered within the ectatic stroma (case 4). It was thickened in all cases. It was also partially or totally infiltrated by microvacuoles scattered within is collagen nodules. Endothelial cells when present were quite normal except in case 4 where they were noted to have abnormally dilated mitochondrias.

\section{Discussion}

The five reported cases had the same clinical appearance which consisted of progressive marginal corneal thinning and ectasia. There were no inflammatory signs in three cases. Repeated episodes of inflammation led to perforation in case 3 while in case 5 some episodic ocular redness was observed. Clinical observation of these cases by the same physician made it possible to distinguish Terrien's disease from the other three corneal marginal conditions with which it could be confused: marginal keratoconus (marginal pellucid degeneration), keratoglobus and Mooren's ulcer.

All cases had paralimbal corneal thinning with more or less ectasia, interstitial vascularisation, annular lipid accumulation in the inner ring of the thinning and distortion of the corneal curvature producing severe against the rule astigmatism. Systemic investigations revealed diabetes in two cases and an alcohol/ tobacco intoxication in one case. There were no signs of any rheumatic or collagen diseases, nor any signs of an acquired immune syndrome. There was no special ocular history in these five cases. All the patients conformed to the classical description of Terrien's disease as discussed by Austin et al. ${ }^{1}$

Although Terrien's disease can be identified as a clear entity, its nature is still obscure. It is a slowly progressive disease lasting for as long as 30 years in some patients. It is rarely associated with inflammatory episodes except when associated with limbal vasculitis,. ${ }^{9}$

All histological examinations showed corneal stromal thinning, an altered basal lamina and Bowman's membrane, a disorganised corneal stroma with interstitial vascularisation, intense lipid infiltration, a thickened Descemet's membrane and few inflammatory cell infiltrates.

Terrien's disease would be much better understood if it showed a specific ultrastructural abnormality as a specific confirmation of abnormalities.

These 5 cases reports are unusual because they have been treated by six penetrating keratoplasties. This has permitted a study of the lesion throughout the full thickness and over the thinned ectatic periphery.

The most important corneal change is the peripheral thinning which reduces it to half the normal thickness. This is not a specific characteristic as thinning occurs in marginal keratoconus, keratoglobus and Mooren's ulcer. Nevertheless, ultrastructural anomalies can clearly differentiate these three limbic conditions from Terrien's marginal ectasia. Marginal keratoconus has the same structure as classical keratoconus; the stroma is thinned, but the lamellar structure is easily recognised. Even if they are full of fine dense deposits, they never present any vacuoles as was reported in the above cases. Furthermore, Bowman's membrane is characteristically broken, but Descemet's membrane is almost always normal inflammatory cell reaction is encountered only when the cornea has perforated. In keratoglobus, protrusion of the entire corneal surface is also characterised by the absence of inflammatory cells. 
In addition acquired and congenital keratoglobus have different specific signs. ${ }^{11}$ Congenital keratoglobus has obvious anomalies of the stromal structure: absence of Bowman's membrane, quantitative modifications in the ratio of collagen to matrix and Descemet's membrane is profoundly altered. The anatomopathogical changes in Mooren's ulcer, in its acute phase, cannot be confused with Terrien's disease because even healed quiescent Mooren's ulcers show an acute reaction in which inflammatory cells prevail.

The five cases reported here make it possible to differentiate Terrien's disease from other limbal conditions.

The stromal changes found in Terrien's disease consist of dense dispersed deposits which are scattered or grouped within the lamella, and which are found in the peripheral stromal lamella, these vacuoles are an important ultrastructural element which is specific of this condition. They can also be found in the anterior layers of a normal Descemet's membrane or in its fragments in the posterior stromal layers. These multiple sometimes confluent deposits are dissolved by fixation are associated with cholesterol lipidic crystals, (in case 3 ) suggesting a lipid nature.

Soudan III staining was positive in cases 4 and 5 and examination of the first three cases by electron microscopy confirms the first description made by Fine et al. ${ }^{4} \mathrm{~A}$ histochemical study was performed which revealed the lipid nature of these lesions. In cases 4 and 5, specific lipid staining for electron microscopy confirmed the lipid nature of the deposits, which confirms the clinical appearance, However, this is not enough to explain the entire paralimbic degeneration of Terrien's disease.

It is possible that this disease, like some primarily lipidic degeneration consist of a magnification of the degenerative process which leads to arcus senilis. However, Iwamoto et $^{8} l^{8}$ showed in their lamellar specimen study that lipid infiltration occurs together with other lesions and that histochemistry also shows the presence of mucopolysaccharide acid deposits ${ }^{14}$ a substance which is highly eosinophlic, PAS positive or intensively stained by PTH, suggesting a fibrinoid collagen degeneration. ${ }^{9}$ These substances were found in the five cases reported here. Iwamoto et al. pay particular attention to the relationship between the agllutination around the vacuoles. This change suggests a possible disorder which may precede vascularisation, interstitial necrosis or even a degenerative process of collagen. This relation, we noticed in Schnyder cristalline dystrophy, ${ }^{8}$ these infiltrates and lipid crystals insinuate themselves through the structure of or collect around collagen fibrils.

All of these stromal modifications (vacuolar infiltrations, dense microgranular deposits, collagen fibrils agglutination or dissociation, fibrinoid degeneration), suggests an organised degeneration process surrounding the lipidic infiltrates. In our five cases, the process is associated with a mild inflammatory cell infiltration.

Stromal inflammatory cells were particularly observed in case 4 which was excessively evoluted and thinned presenting scattered polynuclear cells within its stroma. It was only in case 3 that we observed numerous macrophages and 'spumous' cells surrounding lipid crystals. It was within these areas that we found some plasma cells.

In the other cases, only few lymphocytes were found in the epithelium. In fact, in none of our five cases could we detect a severe inflammatory cell reaction.

Healing and degenerative signs coexist. They are characterised by fibrocytic activity (dense glycogen cytoplasmic aggretates). Corneal scarring represented by either heterogeneous or long periodic collagen fibres. Vessels were numerous, but the blood vessels were not surrounded by numerous inflammatory cells.

Are these anomalies sufficient to explain the development of Terrien's marginal ectasia? It seems unlikely because rings are frequently observed in moderately altered peripheral cornea, in which dense irregular infiltrate developed an arcus senilis where the stroma is modified or the corneal epithelial is thickened above without any thinning of the cornea. These individuals do not show any ectasia or any particular clinical or anatomical evolution.

Could the lipidic deposits by the starting point of a slow degenerative process leading to subsequent modifications or could there be 
additional factors leading to the modifications mentioned above? Berkowitz et al. ${ }^{2}$ tried to answer these questions by searching for circulating immune complexes in peripheral corneal diseases; Mooren's ulcer, Terrien's disease and corneal ulcers associated with connective tissue diseases.

These authors found no difference in any circulating immune complex between normal patients and patients suffering from Terrien's disease. These studies showed that an immune reaction type III could not play any part in the pathogenesis of the disease. This test suggests that it is possible to differentiate Mooren's ulcers from Terrien's degeneration. However, not everyone agrees with this clear differentation between the two conditions, Austin $^{1}$ stated that inflammatory episodes can be encountered during the evolution of Terrien's degeneration and Binder et $a l^{3}$ reported a case of bilateral marginal ulcer which could be easily confused with Terrien's disease. However this case of marginal ectasia was quite different from the five cases reported in this paper. Its anatomico-pathological description shows an inflammatory necrosis of the superficial layers of the cornea while the posterior layers as well as Descemet's membrane were normal. This is like a Mooren's ulcer and is clearly different from the changes we and others have found in Terrien's disease. Nevertheless, it is interesting to consider the views of Binder et al. which refer to a possible similarity between the mechanisms which cause the lesions observed in these two non-infectious affections of the corneal limbus. Although the clinical appearances are similar they cannot be the result of the same disease. In Mooren's ulcer, the presence of immunoglobulins, circulating antibodies and immune complex leads to an active inflammatory lesion. All of these manifestations are absent in Terrien's disease, which in typical form is slowly progressive and ectatic giving rise to slow interstitial necrosis of the disorganised corneal stroma resulting in peripheral thinning.

However, this process is similar to that observed in certain corneal inflammatory lesions, especially herpes, in which there is sometimes stromal thinning. We have found lymphocytes and plasma albumin in our cases so it is possible that inflammatory episodes could play a part in the necrosis particularly as we have one patient who developed kerotosonus and Terrien's disease. The role of the lipid is uncertain. Most authors believe that the excess lipid is only secondary to the interstitial vacuolisation of the thinned area. It is usually present in arcus senilis but here it is not associated with a corneal thinning. However, it is possible that it is one of the promoting factors of lysis and disorganisation of corneal conjunctival tissue leading to corneal peripheral thinning. Lipid deposition is a constant feature of Terrien's disease and its presence in excess cannot be ignored.

At present it appears that the ectactic changes in Terrien's disease are the terminal phase of a condition characterised by structural weakness of the peripheral corneal stroma which is associated with mild inflammatory episodes and the secondary desposition of lipid in the adjacent cornea and conjuctiva.

\section{References}

${ }^{1}$ Austin P and Brown SI: Inflammatory Terrien's marginal corneal disease. Am J Ophthalmol 1981, 92: 189-92.

${ }^{2}$ Berkowitz PJ, Arenssen JJ, Felberg NT, Laibson PR: Presence of circulating immune complexes in patients with peripheral corneal disease. Arch Ophthalmol 1983, 101: 242-5.

${ }^{3}$ Binder P, Zavala EY, Stainer GA: Non-infectious peripheral corneal ulceration: Mooren's ulcer or Terrien's marginal degeneration. Ann Ophthalmol 1982, 14: 425-532.

${ }^{4}$ Fine BS, Thownsend WM, Zimmerman LE, Lashkari MH: Primary lipidal degeneration of the cornea. Am J Ophthalmol 1974, 78: 12-23.

${ }^{5}$ François J: La dégénérescence marginale de la cornée. Arch Ophthalmol 1936, 53: 616.

${ }^{6}$ Fuchs E: Ueber Randsklerose und Randa trophie der Hornhant. Albrecht v Graefe's Arch Klin Ophthalmol 1901, 52: 317.

${ }^{7}$ Fuchs E: Ueber senile Randatrophie der Hornhant. Albrecht v Graefe's Arch Klin Ophthalmol 1915, 89: 386.

${ }^{8}$ Hoang-Xuan T, Pouliquen Y, Savoldelli M, Gasteau J: Dystrophie cristalline de Schnyder. $J \mathrm{Fr}$ Ophtalmol 1985, 8: 735-42.

${ }^{9}$ Iwamoto T, De Voe AG, Farris F: Electron microscopy in cases of marginal degeneration of the cornea. Invest Ophthalmol 1972, 11: 4, 241-57.

${ }^{10}$ Lauber H: Ueber periphere Hornhant ektasie. Klin Monatsbl Augenheilkd 1905, 43: 382.

${ }^{11}$ Pouliquen Y, Espinasse MA, Dhermy P, Savoldelli M: Le kératoglobe. J Fr Ophtalmol 1985, 8: 43-54.

${ }^{12}$ Pouliquen Y, Renard G, Savoldelli M: Keratoconus associated with Terrien's marginal degeneration. Acta Ophthalmologica 1988 (sous presse).

${ }^{13}$ Savino DF, Fine BS, Alldredge OC: Primary lipidic degeneration of the cornea. Cornea 1988, 5: 191-8. 
${ }^{14}$ Suveges L, Levai G, Alberth B: Pathology of Terrien's disease. Histochemical and electron microscopic study. Am J Ophthalmol 1972, 74: 1191-200.

${ }^{15}$ Terrien F: Dystrophie marginale symétrique des deux cornées. Arch Ophtalmol 1900, 20: 12.
${ }^{16}$ Terrien F: La dystrophie marginale ectatique de la cornée. In Semeiologie oculaire : la calotte cornéosclérale. Masson, pp 167-9, 1923.

${ }^{17}$ Trantas A: Ectasie périphérique de la cornée de Terrien (keratoleptensis marginal). Clin Ophtalmol 1925, 14: 621 . 